Pacific Journal of Mathematics

GEN POWER METHODS 


\title{
INCLUSION RELATIONS BETWEEN POWER METHODS OF LIMITATION
}

\author{
ABrahaM Ziv
}

Let $p(x)=\sum p_{k} x^{k}$ be a power series with $p_{k}(k=0,1, \cdots)$ complex numbers and $0<\rho_{p} \leqq \infty$ its radius of convergence, and assume that $P(x) \neq 0$ for $0 \leqq \alpha_{p} \leqq x<\rho_{p}$. The power method of limitation, $P$, is defined by

$$
\lim _{p} s=\lim _{x \rightarrow p^{-}} \sum_{k=0}^{\infty} p_{k} s_{k} x^{k} / P(x) \quad(x \text { real })
$$

(provided the series converges in $\left[\alpha_{p}, \rho_{p}\right)$ and the limit exists and is finite). Abel and Borel methods are the best known power methods. In this article inclusion relations between two power methods are investigated. Several theorems are proved, which lead to necessary and sufficient conditions, for inclusion, that are correct under some fairly moderate restrictions.

1. Introduction. Let $P(x)=\sum p_{k} x^{k}$ be a power series with $p_{k}(k=0,1, \cdots)$ complex numbers and $0<\rho_{p} \leqq \infty$ its radius of convergence, and assume that $P(x) \neq 0$ for $0 \leqq \alpha_{p} \leqq x<\rho_{p}$. The power method of limitation, $P$ (see Włodarski [19] and Birkholc [2]), is defined by

$$
\lim _{p} s=\lim _{x \rightarrow \rho_{p^{-}}} \sum_{k=0}^{\infty} p_{k} s_{k} x^{k} / P(x) \quad(x \text { real })
$$

(provided the series convergences in $\left[\alpha_{p}, \rho_{p}\right.$ ) and the limit exists and is finite).

The power method $Q$ is defined analogously by $Q(x)=\sum q_{k} x^{k}$ and parameters $\alpha_{q}, \rho_{q}$.

The best known power methods are the Abel method and the Borel exponential method. Other power methods which appear in the literature are $A_{\lambda}, L$ and $(B, a, b)$ (for more details see next section).

We are concerned here with inclusion relations of the form $P \subseteq Q$. There are several results in the literature in this direction. Thus, Borwein proved (see [4], [5] and [8]) that $A_{\lambda} \subseteq A_{\mu}$, $A_{\mu} \nsubseteq A_{\lambda}$ provided $-1<\mu<\lambda$, that $A_{\lambda} \subseteq L, L \nsubseteq A_{\lambda}$ provided $\lambda>-1$ and that $(B, a, \beta) \leqq(B, a, b)$ provided $a>0,-\infty<\beta \leqq b<+\infty$.

Other results, obtained by Borwein [4], [8] and Hoischen [12], are of a more general nature. Both authors investigated inclusion relations between power methods whose coefficients, $\left\{p_{k}\right\},\left\{q_{k}\right\}$, are assumed, a priori, to be related by some particular cases of the relation 


$$
\begin{gathered}
q_{k}=p_{k} \int_{0}^{r} \tau^{k-i} d \psi(\tau), \quad 0<r<\infty, i \in\{0,1, \cdots\}, \quad k=n, n+1, \cdots, \\
\int_{0}^{r}|d \psi(\tau)|<\infty .
\end{gathered}
$$

Assuming some more restrictive conditions (like $p_{k} \geqq 0$ or $p_{k} \neq 0$ or others) Borwein gets sufficient conditions and Hoischen necessary and sufficient conditions for inclusion.

In this article we are able to discuss the problem in greater generality. The single essential restriction which still remains necessary is:

$$
\sum_{k p_{k} \neq 0} 1 / k=\infty .
$$

The main tools which make this discussion possible are taken from [21].

It seems that the problem is not simple enough to be solved by one or two theorems. A broader kind of investigation is needed. Actually it comes out that the case of power methods with finite radius of convergence should be separated from the case of infinite radius of convergence. The discussion of the first case provides results which are simpler to formulate and are more satisfactory.

The forthcoming results include, in particulr, necessary conditions, for inclusion, some combinations of which turn out to be also sufficient. So, necessary and sufficient conditions can be formulated, with (1.1) being the only pre-assumed restriction. Those conditions seem to be slightly complicated if $\rho_{p}=\infty$; so they are simplified for some restricted cases, where all the additional restrictions are sufficiently general to be automatically satisfied if $P$ and $Q$ are both regular power methods.

Few of the theorems are applied later to the above mentioned examples of power methods (all of which are regular) yielding some results of interest. Necessary and sufficient conditions for each of the inclusions $P \subseteq A, A \subseteq Q, P \subseteq B, B \subseteq Q$ (where $A, B$ are the Abel and Borel methods and $P, Q$ are some other power methods) are obtained as corollaries.

\section{Definitions and statement of results.}

\subsection{A definition and a convention.}

2.1.1. Power methods of limitation. Let $P(x)=\sum p_{k} x^{k}$, with complex coefficients, $p_{k}(k=0,1, \cdots)$, and radius of convergence 
$0<\rho_{p} \leqq \infty$, be some power series such that $P(x) \neq 0$, for $\alpha_{p} \leqq x<\rho_{p}$, where $0 \leqq \alpha_{p}<\rho_{p}$ is some real constant. A sequence of complex numbers $s=\left\{s_{k}\right\}$ is said to be $P$-convergent to $\sigma$ if $\sum p_{k} s_{k} x^{k}$ is convergent for all $x \in\left[\alpha_{p}, \rho_{p}\right)$ and

$$
\lim _{x \rightarrow \rho_{p^{-}}} T_{p}(s, x)=\sigma, \quad T_{p}(s, x)=\sum_{k=0}^{\infty} p_{k} s_{k} x^{k} / P(x) \quad(x \text { real }) .
$$

$T_{p}(s, x)$ is called the P-transform of $s$ and $\sigma$ its P-limit. $\sigma$ is denoted also by $\lim _{p} s$. By $c_{p}$ we denote the field of the method $P$, i.e. the set of all complex sequences which are $P$-convergent to a finite limit. $\quad c_{p}^{(0)}$ denotes the set of complex sequences which are $P$-limitable to zero, and $m_{p}$ the set of all complex sequences whose $P$-transform exists and is bounded in $\left[\alpha_{p}, \rho_{p}\right)$.

In analogy with $P$ the power method $Q$ is defined by the series $Q(x)=\sum q_{k} x^{k}$ and parameters $\alpha_{q}, \rho_{q}$. The $Q$-transform of a sequence $s$ and its $Q$-limit are denoted by $T_{q}(s, x), \lim _{q} s$. The field of $Q$ and the other related sets are denoted by $c_{q}, c_{q}^{(0)}, m_{q}$.

We say that $P \subseteq Q$ (i.e. $P$ is included in $Q$ ) if $c_{p} \leqq c_{q}$ and $P, Q$ are consistent (i.e. $\lim _{q} s=\lim _{p} s$ for all $s \in c_{p}$ ).

In many of the results of this paper, $P$ is required to satisfy the additional condition

$$
\sum_{k p_{k} \neq 0} 1 / k=\infty .
$$

The following are examples of well known power methods:

A-Abel's method: $P(x)=1 /(1-x), \alpha_{p}=0, \rho_{p}=1$.

$B$-Borel's exponential method: $P(x)=e^{x}, \alpha_{p}=0, \rho_{p}=\infty$.

$A_{\lambda}$-Abel-type methods: $P(x)=(1-x)^{-1-\lambda}, \lambda>-1, \alpha_{p}=0, \rho_{p}=1$ (see Jakimovski [1] and Borwein [5]).

$L$ or $A_{-1}$-Logarithmic method: $\quad P(x)=\log [1 /(1-x)], \quad \alpha_{p}>0$, $\rho_{p}=1$ (see Borwein [6]).

$(B, a, b)$-Borel-type methods: $P(x)=\sum_{k=N}^{\infty} x^{k} / \Gamma(a k+b) \sim a^{-1} x^{(1-b) / a} e^{x^{1 / a}}$ $(x \rightarrow+\infty), a>0,-\infty<b<+\infty, a N+b>0, \alpha_{p}>0, \rho_{p}=\infty$ (see Borwein [8]).

2.1.2. A convention about functions of bounded variation. Every complex valued function whose variation is bounded in some finite or infinite interval is assumed throughout to be continuous to the right at all points of this interval, with the possible exception of the interval's ends.

2.2. Theory which is restricted only by condition (2.1.1). 
REMARK 2.2.1. It should be observed that if $P(x)$ is a polynomial then every sequence is $P$-convergent. This is, then, a trivial case. For this reason it is assumed throughout that both $P(x)$ and $Q(x)$ are not polynomials.

REMARK 2.2.2. If $c_{p}^{(0)} \subseteq m_{q}$ then the set $J=\left\{k \mid p_{k}=0, q_{k} \neq 0\right\}$ is finite.

2.2.3. Definition of $r_{p q}$. We define

$$
r_{p q}=\varlimsup_{k \rightarrow \infty}\left|q_{k} / p_{k}\right|^{1 / k},
$$

where $k$ is considered, in the limiting process, only if $p_{k} \neq 0$.

THEOREM 2.2.4. Let (2.1.1) be satisfied. If $c_{p}^{(0)} \cong m_{q}$ then each of the following must be satisfied:

(i ) $0<r_{p q}<\infty$

(ii) $\rho_{q} r_{p q}=\rho_{p}$

(iii) The limit

$$
\lim _{k \rightarrow \infty} q_{k} /\left(p_{k} r_{p q}^{k}\right) \quad\left(k \text { is considered only if } p_{k} \neq 0\right)
$$

exists and is finite.

COROLLARY 2.2.5. Let (2.1.1) be satisfied. If $e_{p}^{(0)} \cong m_{q}$ then either $\rho_{p}<\infty$ and $\rho_{q}<\infty$ or $\rho_{p}=\rho_{q}=\infty$.

THEOREM 2.2.6. Let (2.1.1) be satisfied and assume that $\rho_{p}<\infty$. If $c_{p}^{(0)} \subseteq m_{q}$ then a function $\phi$ of bounded variation and constants $0<\theta<1, m \in\{0,1, \cdots\}$ exist, which satisfy:

$$
\begin{gathered}
q_{k}=p_{k} r_{p q}^{k}\left(\int_{0}^{1} \tau^{k} d \dot{\phi}(\tau)+O\left(\theta^{k}\right)\right) \quad(k \rightarrow \infty, k=m, m+1, \cdots) \\
\int_{0}^{1}\left|P\left(x r_{p q} \tau\right)\right||d \phi(\tau)|=O(Q(x)) \quad\left(x \rightarrow \rho_{q}^{-}, x \text { real }\right) .
\end{gathered}
$$

THEOREM 2.2.7. Let (2.1.1) be satisfied and assume that $\rho_{p}<\infty$. If $c_{p} \subseteq c_{q}$ then the limit $1 / Q\left(\rho_{q}-0\right)$ must exist and be finite, unless $Q(x) \equiv \kappa P\left(x r_{p q}\right), \kappa \neq 0$ (in which case $P$ and $Q$ are trivially equivalent). If, further, $P \subseteq Q$ (and $Q(x) \not \equiv \kappa P\left(x r_{p q}\right)$ ) then the said limit must be zero.

REMARK 2.2.8. Theorem 2.2.7 cannot be extended to the case $\rho_{p}=\infty$. In fact an example is given in Section 3.1.15 of two essentially different power methods, $P, Q$, with $\rho_{p}=\rho_{q}=\infty$ which satisfy $P \subseteq Q$, while the limit $1 / Q(+\infty)$ does not exist. 
THEOREM 2.2.9. Let (2.1.1) be satisfied and assume that $\rho_{p}=\infty$. If $c_{p}^{(0)} \subseteq m_{q}$ then there exist: a function $\phi$, whose variation is bounded in $[\varepsilon, 1]$, for every $\varepsilon>0$, constants $0 \leqq R<+\infty, u\left(\alpha_{q}<u<+\infty\right.$, $\left.u r_{p q}>\alpha_{p}\right), m \in\{0,1, \cdots\}$ and a matrix $\left(e_{x k}\right)(u \leqq x<+\infty, k=0,1, \cdots)$ such that

( i ) $q_{k}=p_{k} r_{p q}^{k} \int_{u ; x}^{1} \tau^{k} d \phi(\tau)+Q(x) e_{x k} / x^{k}$, $(u \leqq x<+\infty, k=0,1, \cdots)$.

(ii ) $\int_{u \mid x}^{1}\left|P\left(x r_{p q} \tau\right)\right||d \phi(\tau)|=O(Q(x)), \quad(x \rightarrow+\infty, x$ real $)$.

(iii) $\begin{cases}\left|e_{x k}\right| \leqq R\left|p_{k}\right| u^{k} r_{p q}^{k}, & (u \leqq x<+\infty, k=m, m+1, \cdots) \\ \left|e_{x k}\right| \leqq R, & (u \leqq x<+\infty, k=0,1, \cdots) .\end{cases}$

If, further, $c_{p} \subseteq c_{q}$ and we denote

$$
e_{x k}^{\prime}=e_{x k}-\left[p_{k} u^{k} r_{p q}^{k} / P\left(u r_{p q}\right)\right] \sum_{\imath=0}^{\infty} e_{x \imath} \quad(u \leqq x<+\infty, k=0,1, \cdots)
$$

then, in addition,

(iv) The limits

$$
e_{k}^{\prime}=\lim _{x \rightarrow+\infty} e_{x k}^{\prime} \quad(x \text { real, } k=0,1, \cdots)
$$

all exist and are finite.

( v) The limit

$$
\gamma(T)=\lim _{x \rightarrow+\infty} \int_{u \mid x}^{1} P\left(x r_{p q} \tau\right) T(x \tau) d \phi(\tau) / Q(x) \quad(x \text { real })
$$

exists and is finite, for every function $T(t)$, which is continuous in the interval $[u,+\infty)$, vanishes at its left end and has a finite limit $T(+\infty)$.

If, further, $P \subseteq Q$ then, in addition,

(iv) $e_{k}^{\prime}=0 \quad(k=0,1 \cdots)$

(v)' $\gamma(T)=T(+\infty)$.

REMARK 2.2.10. It is of interest to note that $\phi$ is not constant in $[1-\delta, 1]$ for any $\delta>0$. This is true in Theorem 2.2.6 as well as in Theorem 2.2.9. In Theorem 2.2.6 $\phi$ is uniquely determined (up to an additive constant) in $[\theta, 1]$ and in Theorem 2.2 .9 it is uniquely determined in $(0,1]$.

THEOREM 2.2.11. Assume that $\rho_{p}<\infty$. If $0<r_{p q}<\infty, \rho_{q} r_{p q}=$ $\rho_{p}, \quad 1 / Q\left(\rho_{q}-0\right)=0$ and (2.2.1), (2.2.2) are satisfied, with $\phi$ of bounded variation and some constants $0<\theta<1, m \in\{0,1, \cdots\}$ then $P \subseteq Q$.

Corollary 2.2.12. Assume that (2.1.1) is satisfied, $\rho_{p}<\infty$ and $1 / Q\left(\rho_{q}-0\right)=0$. If $c_{p}^{(0)} \cong m_{q}$ then $P \subseteq Q$. 
ThEOREM 2.2.13. Assume that $\rho_{p}=\infty$. If $0<r_{p q}<\infty$ and conditions (i), (iii), (iv), (v) of Theorem 2.2 .9 are satisfied, then $c_{p} \subseteq c_{q}$. If conditions (iv)', (v)' of Theorem 2.2 .9 are satisfied also then $P \subseteq Q$.

REMARK 2.2.14. In Theorems 2.2.11 and 2.2.13 it need not be pre-assumed that $r_{p q}$ satisfies Definition 2.2.3. The rest of the requirements mentioned in the theorems suffice.

2.3. Restricted results. Power methods $P$ with $p_{k} \geqq 0(k=0$, $1, \cdots)$ were investigated more than others in the past. Therefore they are of special interest, and an attempt to speciallize some theorems for them is worthwhile. It happens that the results of this attempt gave rise to theorems which are applicable to conservative and regular power methods in general, and therefore we begin with the characterization of these kinds of methods:

2.3.1. Definition of $\hat{P}(x)$. Given $P(x)=\sum p_{k} x^{k}$, we define

$$
\hat{P}(x)=\sum_{k=0}^{\infty}\left|p_{k}\right| x^{k}, \quad\left(\alpha_{p} \leqq x<\rho_{p}\right) .
$$

Obviously $|P(x)| \leqq \hat{P}(x), \alpha_{p} \leqq x<\rho_{p}$.

THEOREM 2.3.2. $P$ is conservative if and only if

(i) A constant $L>0$ exists such that

$$
L \hat{P}(x) \leqq|P(x)| \leqq \hat{P}(x) \quad\left(\alpha_{p} \leqq x<\rho_{p}\right)
$$

and

(ii) The limit $1 / P\left(\rho_{p}-0\right)$ exists and is finite. $P$ is regular if and only if, in addition

(ii $)^{\prime} \quad 1 / P\left(\rho_{p}-0\right)=0$ and

(iii) $P(x)$ is not a polynomial.

REMARK 2.3.3. It should be observed that (iii) of Theorem 2.3.2 is automatically satisfied if $\rho_{p}<\infty$ and that (i) $\Rightarrow$ (ii)' in case $\rho_{p}=\infty$ (unless $P(x) \equiv$ const.).

THEOREM 2.3.4. Let $P$ be conservative and satisfy (2.1.1). If $c_{p}^{(0)} \subseteq m_{q}$ then the limit

$$
\lim _{x \rightarrow \rho_{q^{-}}} Q(x) / P\left(x r_{p q}\right) \quad(x \text { real })
$$

exists and is finite. If, further, $P$ is regular and $\rho_{p}=\infty$ then in addition 


$$
\lim _{x \rightarrow+\infty}|Q(x) / P(x r)|=\left\{\begin{array}{ll}
+\infty, & 0<r<r_{p q} \\
0, & r_{p q}<r<+\infty
\end{array}, \quad(x \text { real })\right.
$$

TheOREM 2.3.5. Let (2.1.1) be satisfied and assume that $\rho_{p}<\infty$ and that the limit $1 / P\left(\rho_{p}-0\right)$ exists and is finite. If $0<r_{p q}<\infty$, $\rho_{q} r_{p q}=\rho_{p}$, the limit $1 / Q\left(\rho_{q}-0\right)$ exists and is finite and (2.2.1), (2.2.2) are satisfied, with some $\phi$ of bounded variation, $0<\theta<1$ and some $m \in\{0,1, \cdots\}$ then $c_{p} \subseteq c_{q}$.

2.3.6. Definition of $e, e^{(l)}, U$. We denote $e=(1,1,1, \cdots)$ and $e^{(l)}=(0, \cdots, 0,1,0, \cdots)$, where the single 1 is at the $l$ th place. Also $U=\{e\} \cup\left\{e^{(l)} \mid l=0,1, \cdots\right\}$.

Conditions under which $U$ forms a fundamental set in $c_{p}$ are to be found in [21] (see also Włodarski [19] and Birkholc [2], [3]). In particular $U$ is foundamental in the fields of the Abel and Borel methods (see Zeller [20] and Ryll-Nardzewski [15]).

THEOREM 2.3.7. Assume that $\rho_{p}=\infty$ and that either $U$ is fundamental in $c_{p}$ or $Q(\varepsilon x) / Q(x) \rightarrow 0(x \rightarrow+\infty, x$ real $)$ for every sufficiently small $\varepsilon>0$. If $0<r_{p q}<\infty$ and conditions (i), (ii), (iii) of Theorem 2.2.9 are satisfied then $P \subseteq Q$.

REMARK 2.3.8. Theorem 2.3.7 is applicable to the case that $Q$ is regular for in this case $Q(\varepsilon x) / Q(x) \rightarrow 0$ for every $0<\varepsilon<1$.

Corollary 2.3.9. Let (2.1.1) be satisfied and assume that $\rho_{p}=\infty$ and that either $U$ is fundamental in $c_{p}$ or $Q(\varepsilon x) / Q(x) \rightarrow 0(x \rightarrow+\infty$, $x$ real) for every sufficiently small $\varepsilon>0$. If $c_{p}^{(0)} \leqq m_{q}$ then $P \subseteq Q$.

The following theorem provides an easy means of producing examples of inclusions between non-regular power methods, with $\rho_{p}=\infty$.

THEOREM 2.3.10. Assume that $\rho_{p}=\infty$ and that the limit $P(+\infty)$ exists and is finite. If

$$
q_{k}=p_{k} r^{k} \int_{0}^{1} \tau^{k} d \phi(\tau) \quad(k=0,1, \cdots),
$$

where $0<r<\infty$ and $\phi$ is of bounded variation, then $\rho_{q}=\infty$ and the limit $Q(+\infty)$ exists and is finite. If, further, $Q(+\infty) \neq 0$ then $c_{p} \subseteq c_{q} . \quad$ If, in addition, $P(0)[\phi(0+)-\phi(0)]=0$ then $P \subseteq Q$.

2.4. Examples. In this section we present some results of applying the general theorems of the previous sections to particular power methods. 
2.4.1. A simple test of inclusion. Let us investigate among the particular power methods defined at the end of $\S 2.1 .1$, which pairs may satisfy an inclusion relation. An easy preliminary check can be performed by application of Theorem 2.2.4 and its Corollaries 2.2.5 and 2.3.4.

Thus, calculation of $r_{p q}$ for the relevant pairs of methods immediately excludes, by (i) of Theorem 2.2.4, the possibility of inclusion between $A_{\lambda}$ or $L$ and $(B, a, b)$. It also proves the impossibility of inclusion between $(B, a, b)$ and $(B, \alpha, \beta)$ in case $a \neq \alpha$. (In fact no direct calculation of $r_{p q}$ is needed. One can use Corollary 2.2.5 and Theorem 2.3.4 instead.)

No further conclusions can be drawn by (i) of Theorem 2.2.4. However (iii) of this theorem or, its corollary, the first part of Theorem 2.3.4, provides a finer test and its application shows that $L \nsubseteq A_{\mu} \nsubseteq A_{\lambda}$ if $\lambda>\mu>-1$ and that $(B, a, b) \nsubseteq(B, a, \beta)$ if $a>0$, $-\infty<\beta<b<+\infty$. So after a complete check with Theorem 2.2.4 it seems that the only possible inclusions are $A_{\lambda} \leqq A_{\mu} \leqq L(\lambda \geqq \mu>$ $-1)$ and $(B, a, \beta) \leqq(B, a, b) \quad(a>0,-\infty<\beta \leqq b<+\infty)$. Those inclusions are, in fact, known to be valid and were proved by $D$. Borwein (see [4] and [8]) using methods which could be interpreted as applications of Theorems 2.2.11 and 2.3.7.

The results of the previous sections make it possible to solve completely certain inclusion problems. As examples we formulate necessary and sufficient conditions for the inclusions $A \subseteq Q, P \subseteq A$, $B \subseteq Q, P \subseteq B$ where $A, B$ are Abel and Borel methods and $P, Q$ are any power methods (not restricted in any sense).

In the following $c_{a}, c_{b}^{(0)}, m_{b}$ denote, respectively, the field of the Abel method and the appropriate sets which are related to Borel method.

COROLlaRY 2.4.2. In order that $c_{a} \leqq c_{q}$ it is necessary and sufficient that the following is satisfied: $\rho_{q}<\infty$, the limit $1 / Q\left(\rho_{q}-0\right)$ exists and is finite and

where

$$
q_{k} \rho_{q}^{k}=\int_{0}^{1} \tau^{k} d \phi(\tau)+O\left(\theta^{k}\right) \quad(k \rightarrow \infty)
$$

$$
\begin{array}{r}
\int_{0}^{1}|d \phi(\tau)|<\infty, \quad 0<\theta<1, \quad \int_{0}^{1}\left[\rho_{q} /\left(\rho_{q}-x \tau\right)\right]|d \phi(\tau)| \\
=O(Q(x)) \\
\left(x \rightarrow \rho_{q}^{-}, x \text { real }\right) .
\end{array}
$$

The same, with the additional condition $1 / Q\left(\rho_{q}-0\right)=0$, is necessary and sufficient for $A \subseteq Q$. 
COROLlaRY 2.4.3. In order that $c_{p} \subseteq c_{a}$ it is necessary and sufficient that the following is satisfied: $\rho_{p}<\infty, p_{k} \neq 0$, for all sufficiently large $k$, and

$$
1 /\left(p_{k} \rho_{p}^{k}\right)=\int_{0}^{1} \tau^{k} d \phi(\tau)+O\left(\theta^{k}\right) \quad(k \rightarrow \infty)
$$

where

$$
\begin{aligned}
\int_{0}^{1}|d \phi(\tau)|<\infty, \quad 0<\theta<1, \quad \int_{0}^{1}\left|P\left(x \rho_{p} \tau\right)\right||d \phi(\tau)| & =O[1 /(1-x)], \\
& (x \rightarrow 1-, x \text { real }) .
\end{aligned}
$$

The same is necessary and sufficient for $P \subseteq A$.

CoROLLARY 2.4.4. In order that $c_{b}^{(0)} \leqq m_{q}$ it is necessary and sufficient that

$$
0<r_{b q}=\varlimsup_{k \rightarrow \infty}\left|q_{k} k !\right|^{1 / k}<+\infty
$$

and that

$$
q_{k}=\left(r_{b q}^{k} / k !\right) \int_{u \mid x}^{1} \tau^{k} d \phi(\tau)+Q(x) e_{x k} / x^{k}, \quad(u \leqq x<+\infty, k=0,1, \cdots)
$$

where

$$
\begin{gathered}
\int_{u \mid x}^{1}|d \phi(\tau)|<\infty, \quad(u \leqq x<+\infty) \\
\int_{u \mid x}^{1} e^{x r_{b q} \tau}|d \phi(\tau)|=O(Q(x)), \quad(x \rightarrow+\infty, x \text { real }) \\
\left|e_{x k}\right| \leqq R r_{b q}^{k} u^{k} / k ! \quad(u \leqq x<+\infty, k=m, m+1, \cdots) \\
\left|e_{x k l}\right| \leqq R, \quad(u \leqq x<+\infty, k=0,1, \cdots) \\
\alpha_{q}<u<+\infty, \quad 0 \leqq R<+\infty, \quad m \in\{0,1, \cdots\} .
\end{gathered}
$$

The same is necessary and sufficient for $B \cong Q$.

CoROLlary 2.4.5. In order that $c_{p}^{(0)} \subseteq m_{b}$ it is necessary and sufficient that the following is satisfied: $p_{k} \neq 0$ for all sufficiently large $k$,

$$
0<r_{p b}=\varlimsup_{k \rightarrow \infty}\left|p_{k} k !\right|^{-1 / k}<\infty
$$

and

$$
1 / k !=p_{k} r_{p b}^{k} \int_{u / x}^{1} \tau^{k} d \phi(\tau)+e^{x} e_{x k} / x^{k}, \quad(u \leqq x<+\infty, k=0,1, \cdots)
$$




$$
\begin{gathered}
\int_{u ! x}^{1}|d \phi(\tau)|<\infty \\
\left|e_{x k}\right| \leqq R\left|p_{k}\right| u^{k} r_{p b}^{k}, \quad(u \leqq x<+\infty, k=m, m+1, \cdots) \\
\left|e_{x k}\right| \leqq R, \quad(u \leqq x<+\infty, k=0,1, \cdots) \\
\int_{u \mid x}^{1}\left|P\left(x r_{p b} \tau\right)\right||d \phi(\tau)|=O\left(e^{x}\right) \quad(x \rightarrow+\infty, x \text { real }) \\
0<u<+\infty, \quad 0 \leqq R<+\infty, \quad m \in\{0,1, \cdots\} .
\end{gathered}
$$

The same is necessary and sufficient for $P \subseteq B$.

\section{Proofs,}

3.1. Proofs of the results in $\$ 2.2$.

3.1.1. Proof of Remark 2.2.2. Define

$$
s_{k}=\left\{\begin{array}{ll}
0 & , \quad k \notin J \\
1 /\left(q_{k} a^{k}\right), & k \in J
\end{array}, \quad \alpha_{q}<a<\rho_{q}, \quad k=0,1, \cdots .\right.
$$

Obviously $s \in c_{p}^{(0)}$. However, $\sum q_{k} s_{k} a^{k}$ would not converge if $J$ is infinite. Hence $s \notin m_{q}$, in such a case, which means that $c_{p}^{(0)} \subseteq m_{q}$ cannot hold.

In the forthcoming proofs we use several results, which are cited below. The first is a generalization, due to $R$. Trautner [16] of a well known theorem of J. G. Mikusinski [14]:

TrautneR's Theorem 3.1.2 (R. Trautner [16]). Let

$$
\mu_{k}=\int_{0}^{1} \tau^{k} d \chi(\tau), \quad \int_{0}^{1}|d \chi(\tau)|<\infty \quad(k=0,1, \cdots)
$$

be Hausdorff moments. If

$$
\mu_{k_{i}}=O\left(r^{k_{i}}\right) \quad\left(i \rightarrow \infty, 0<r<1, k_{i} \uparrow \infty, k_{i} \text { natural numbers }\right)
$$

then either $\chi=$ const. in $[r, 1]$ or $\sum_{i} 1 / k_{i}<\infty$.

An immediate corollary of Trautner's theorem is:

LEMMA 3.1.3. Let (2.1.1) be satisfied and assume that $g$ is a function of bounded variation in $\left[\alpha_{p}, \rho_{p}\right)$, which is constant in $\left[r^{\prime}, \rho_{p}\right)$, for some $r^{\prime}<\rho_{p}$. If

$$
\begin{aligned}
\left|P_{k} \int_{\alpha_{p}}^{\rho_{p^{-}}}\left[t^{k} / P(t)\right] d g(t)\right| \leqq R\left|p_{k}\right| r^{k}, \quad \alpha_{p}<r<\rho_{p} \\
\\
(k=m, m+1, \cdots)
\end{aligned}
$$


then $g=$ const. in $\left[r, \rho_{p}\right)$.

The rest of the cited results are taken from A. Ziv [21]:

LeMmA 3.1.4 (A. Ziv [21]). Let (2.1.1) be satisfied and let $n \in\{0,1, \cdots\}, \varepsilon>0, \alpha_{p} \leqq r<\rho_{p}, S_{0}, S_{1}, \cdots S_{n-1}$ be given. Assume that $T(x)$ is any function, continuous in $\left[r, \rho_{p}\right)$, having a finite limit $T\left(\rho_{p}-0\right)$ and satisfying

$$
T(r)=\sum_{k=0}^{n-1} p_{k} S_{k} r^{k} / P(r)
$$

and let $\delta(x)>0$ be continuous in $\left[r, \rho_{p}\right)$. There exists a sequence $s \in c_{p}$ which satisfies

$$
\begin{cases}s_{j}=S_{j} \quad(j=0,1, \cdots, n-1) ; & \sum_{k=n}^{\infty}\left|p_{k} s_{l k}\right| r^{k}<\varepsilon \\ \left|T_{p}(s, x)-T(x)\right|<\delta(x) \quad\left(r \leqq x<\rho_{p}\right), & \lim _{p} s=T\left(\rho_{p}-0\right) .\end{cases}
$$

THEOREM 3.1.5 (A. Ziv [21]). Let (2.1.1) be satisfied. If $\sum \beta_{k} s_{k}$ converges for all $s \in c_{p}^{(0)}$ then

$$
\left|\beta_{k}\right| \leqq R\left|p_{k}\right| r^{k}, \quad r<\rho_{p}, \quad k=m, m+1, \cdots, m \in\{0,1, \cdots\} .
$$

3.1.6. Matrix methods of limitation (see Lazić [13] and Ziv [21]). Let $W=\left\{w_{x k}\right\}(x \in I, k=0,1, \cdots)$ be an infinite matrix of complex numbers with $I$ a subset of some topological space. Let $x_{0} \notin I$ be a point of accumulation of $I$, which has a denumerable basis of neighborhoods. A sequence $s=\left\{s_{k}\right\}$ of complex numbers is said to be $W$-ocnvergent to $\sigma$ if its $W$-transform,

$$
T_{w}(s, x)=\sum_{k=0}^{\infty} w_{x k} s_{k},
$$

exists for all $x \in I$ and $\lim _{x \rightarrow x_{0}} T_{w}(s, x)=\sigma(x \in I)$. We denote by $m_{w}$ the set of all sequences whose $W$-transforms exist and are bounded in $I$.

TheOREM 3.1.7 (A. Ziv [21]). Let (2.1.1) be satisfied. If $c_{p}^{(0)} \subseteq m_{w}$ then the matrix $W$ may be decomposed in the form $W=C+D$, where the matrices $C=\left(c_{x k}\right), D=\left(d_{x k}\right)(x \in I, k=0,1, \cdots)$ satisfy $(x \in I, k=0,1, \cdots)$.

(i ) $c_{x k}=p_{k} \int_{\alpha_{p}}^{r_{x}}\left[t^{k} / P(t)\right] d g_{x}(t), \int_{\alpha_{p}}^{r_{x}}\left|d g_{x}(t)\right| \leqq R<\infty, \alpha_{p}<r_{x}<\rho_{p}$,

(ii) $\left|d_{x k}\right| \leqq R\left|p_{k}\right| r^{k}, \quad \alpha_{p}<r<\rho_{p}, \quad(x \in I, k=m, m+1, \cdots$, $m \in\{0,1, \cdots\}) ;\left|d_{x k}\right| \leqq R<\infty(x \in I, k=0,1, \cdots)$. 
$r_{x}, g_{x}$ are independent of $k$ and $R, r, m$ are independent of both $k$ and $x$.

3.1.8. Proof of (i) and (ii) of Theorem 2.2.4. If $c_{p}^{(0)} \subseteq m_{q}$ then $\sum q_{k} s_{k} x^{k}$ converges for all $s \in c_{p}^{(0)}$. Therefore it follows, from Theorem 3.1.5, that

$$
x r_{p q}<\rho_{p} \text { for all } x \in\left[\alpha_{q}, \rho_{q}\right)
$$

which implies that

$$
r_{p q}<\infty, \quad \rho_{q} r_{p q} \leqq \rho_{p} .
$$

Considering only values of $k$ such that $p_{k} \neq 0$ we get

$$
\rho_{p}^{-1} r_{p q}=\left(\varlimsup_{k \rightarrow \infty}\left|p_{k}\right|^{1 / k}\right)\left(\varlimsup_{k \rightarrow \infty}\left|q_{k} / p_{k}\right|^{1 / k}\right) \geqq \varlimsup_{k \rightarrow \infty}\left|q_{k}\right|^{1 / k} ;
$$

so by Remark 2.2.2

$$
r_{p q} / \rho_{p} \geqq 1 / \rho_{q} .
$$

Now we divide the discussion into two cases. First the case $\rho_{q}<\infty$ : In this case (3.1.5) implies-since $r_{p q}<\infty$-that $\rho_{p}<\infty$ and that $r_{p q}>0$. This by (3.1.4) and (3.1.5) completes the proof.

Next assume that $\rho_{q}=\infty$. Had we shown that $r_{p q}>0$ we would get from (3.1.3) that $\rho_{p}=\infty$ which completes the proof. So let us try to reach a contradiction while assuming that $r_{p q}=0$.

The method $Q$ is a matrix method (see $\S 3.1 .6$ ) with $w_{x k}=$ $q_{k} x^{k} / Q(x),\left(x \in\left[\alpha_{q},+\infty\right), k=0,1, \cdots\right)$. The assumption $r_{p q}=0$ means that

$$
\lim _{k \rightarrow \infty}\left|w_{x k} / p_{k}\right|^{1 / k}=0 \quad\left(k \text { is considered only if } p_{k} \neq 0\right) \text {. }
$$

Hence from Theorem 3.1.7 we get

$$
\left|p_{k} \int_{\alpha_{p}}^{r_{x}}\left[t^{k} / P(t)\right] d g_{x}(t)\right|=p_{k} O\left(r^{k}\right), \quad(k \rightarrow \infty)
$$

which implies, by Lemma 3.1.3, that $r_{x}$ need not exceed $r$. Estimating $w_{x k}$ by Theorem 3.1.7, we get, therefore,

$$
\begin{aligned}
\left|q_{k} x^{k} / Q(x)\right| \leqq M\left|p_{k}\right| r^{k}, \quad r<\rho_{p}, \\
\left(\alpha_{q} \leqq x<+\infty, k=m, m+1, \cdots\right)
\end{aligned}
$$

where, $M$ and $r$ are independent of both $x$ and $k$. Let $l \in\{0,1, \cdots\}$ be larger than both $m$ and $k$ and such that $q_{l} \neq 0$ (see Remark 2.2.1). We get

$$
\left|x^{k} / Q(x)\right|=\left|q_{l} x^{l-k}\right|^{-1}\left|q_{l} x^{l} / Q(x)\right| \leqq\left|q_{l} x^{l-k}\right|^{-1} M\left|p_{l}\right| r^{l} \rightarrow 0,(x \rightarrow+\infty)
$$


which leads to the following absurdity:

$$
1=\lim _{x \rightarrow+\infty} \sum_{k=0}^{\infty} q_{k} x^{k} / Q(x)=\sum_{k=0}^{\infty} \lim _{x \rightarrow+\infty} q_{k} x^{k} / Q(x)=0 .
$$

The summation-limitation exchange is permitted because the series is majorized by $\sum M\left|p_{k}\right| r^{k}$ (see (3.1.6)).

REMARK 3.1.9. In view of (i), (ii) of Theorem 2.2.4, it is clear that the variable $x$ of $Q(x)$ may, in cases of inclusion, be scaled to yield $r_{p q}=1, \rho_{q}=\rho_{p}=\rho$. We may also increase either $\alpha_{p}$ or $\alpha_{q}$ to get $\alpha_{q}=\alpha_{p}=\alpha$. It enables us to simplify later proofs by assuming those "normalizing conditions".

The Fundamental Lemma 3.1.10. Let (2.1.1) be satisfied and assume that $r_{p q}=1, \rho_{q}=\rho_{p}=\rho, \alpha_{q}=\alpha_{p}=\alpha$. If $c_{p}^{(0)} \cong m_{q}$ then there exist a function $\chi(t)$, a matrix $e_{x k}$ and constants $R<\infty$, $\alpha<u<\rho, m \in\{0,1, \cdots\}$ such that:

$$
\begin{gathered}
\int_{u \mid x}^{1}|d \chi(\tau)|<\infty, \quad(u \leqq x<\rho) \\
q_{k}=p_{k} \int_{u \mid x}^{1} \tau^{k} d \chi(\tau)+Q(x) e_{x k} / x^{k}, \quad(u \leqq x<\rho, k=0,1, \cdots) \\
|Q(x)|^{-1} \int_{u \mid x}^{1}|P(x \tau)||d \chi(\tau)| \leqq R, \quad(u \leqq x<\rho) \\
\begin{cases}\left|e_{x k}\right| \leqq R\left|p_{k}\right| u^{k}, & (u \leqq x<\rho, k=m, m+1, \cdots) \\
\left|e_{x k}\right| \leqq R, & (u \leqq x<\rho, k=0,1, \cdots) .\end{cases}
\end{gathered}
$$

Proof. Using Theorem 3.1.7 we see that

$$
q_{k} x^{k} / Q(x)=c_{x k}+d_{x k} \quad(\alpha \leqq x<\rho, k=0,1, \cdots)
$$

where $c_{x k}, d_{x k}$ satisfy (i), (ii) of that theorem.

Define $u=r$ (hence $\alpha<u<\rho$ ). Since $r_{p q}=1$ we have from (3.1.11), for all $r=u \leqq x<\rho, \varepsilon>0$ and for $k \rightarrow \infty$,

$$
c_{x k}=p_{k} \int_{\alpha}^{r_{x}}\left[t^{k} / P(t)\right] d g_{x}(t)=q_{k} x^{k} / Q(x)-d_{x k}=p_{k} O\left[(1+\varepsilon)^{k} x^{k}\right] .
$$

Hence, defining $g_{x}=$ const. in $\left[r_{x}, \rho\right)$, we get, from Lemma 3.1.3, that $g_{x}=$ const. in $[x, \rho)$, so

$$
c_{x k}=p_{k} \int_{\alpha}^{x}\left[t^{k} / P(t)\right] d g_{x}(t), \quad(u \leqq x<\rho, k=0,1, \cdots) .
$$

And if we define 


$$
\chi_{x}(\tau)=-Q(x) \int_{x \tau}^{x}[1 / P(t)] d g_{x}(t), \quad(\alpha / x \leqq \tau \leqq 1, u \leqq x<\rho)
$$

we get from (3.1.11)

$$
q_{k}=p_{k} \int_{\alpha \mid x}^{1} \tau^{k} d \chi_{x}(\tau)+Q(x) d_{x k} / x^{k}, \quad(u \leqq x<\rho, k=0,1, \cdots) .
$$

Let $u \leqq x \leqq y<\rho$. From (3.1.12) we get

$$
q_{k}=p_{k} \int_{\alpha / y}^{1} \tau^{k} d \chi_{y}(\tau)+Q(y) d_{y k} / y^{k}
$$

Comparing this with (3.1.12) and using (ii) of Theorem 3.1.7 we get for $k \rightarrow \infty$

$$
p_{k} \int_{\alpha \mid x}^{1} \tau^{k} d\left[\chi_{x}(\tau)-\chi_{y}(\tau)\right]=p_{k} O\left(u^{k} / x^{k}\right), \quad(u \leqq x \leqq y<\rho) .
$$

From Trautner's theorem (see 3.1.2), and because $\chi_{x}(1)=\chi_{y}(1)=0$, we deduce that $\chi_{y}(\tau)=\chi_{x}(\tau)$ whenever $u \leqq x \leqq y<\rho$ and $u / x \leqq \tau \leqq 1$. This enables us to, uniquely, define a function $\chi(\tau)$ in $(u / \rho, 1]$ by:

$$
\chi(\tau)=\chi_{x}(\tau), \quad \tau \in[u / x, 1], \quad(u \leqq x<\rho) .
$$

(3.1.7) follows now immediately from the definition of $\chi_{x}$. Obviously

$$
\begin{aligned}
|Q(x)|^{-1} \int_{u \mid x}^{1}|P(x \tau)||d \chi(\tau)| & =|Q(x)|^{-1} \int_{u \mid x}^{1}|P(x \tau)|\left|d \chi_{x}(\tau)\right| \\
& =\int_{u}^{x}\left|d g_{x}(t)\right| \leqq R .
\end{aligned}
$$

So (3.1.9) is satisfied.

$e_{x k}$ is defined now by (3.1.8). From (3.1.11) and (3.1.8) we get

$$
\begin{aligned}
\left|e_{x k}\right| & =\left|q_{k} x^{k} / Q(x)-p_{k}[Q(x)]^{-1} \int_{u \mid x}^{1}(x \tau)^{k} d \chi_{x}(\tau)\right| \\
& =\left|d_{x k}+p_{k} \int_{\alpha}^{u}\left[t^{k} / P(t)\right] d g_{x}(t)\right| \leqq\left|d_{x k}\right|+\left|p_{k}\right| u^{k} R \sup _{\alpha \leqq t \leqq u}|P(t)|^{-1},
\end{aligned}
$$

which implies (3.1.10), by (ii) of Theorem 3.1.7.

3.1.11. Proof of (iii) of Theorem 2.2.4. By Remark 3.1.9 we may restrict the discussion to the case $r_{p q}=1$. In this case (iii) follows immediately from Lemma 3.1.10. In fact we get $\lim _{k \rightarrow \infty} q_{k} / p_{k}=$ $\chi(1)-\chi(1-0)$.

3.1.12. Proof of Theorem 2.2.6. We insert in Lemma 3.1.10 $u<y<\rho_{q}$ in place of $x$ and define 


$$
\phi(\tau)= \begin{cases}\chi(\tau), & u / y \leqq \tau \leqq 1 \\ \text { const. }, & 0 \leqq \tau \leqq u / y\end{cases}
$$

Then (2.2.1), with $\theta=u / y$, follows immediately from (3.1.8) and 3.1.10). (2.2.2) follows from (3.1.9).

LEMMA 3.1.13.

(a) If in Lemma 3.1.10 we add the assumption $c_{p} \leqq c_{q}$, then the limit

$$
\gamma(T)=\lim _{x \rightarrow \rho-}[Q(x)]^{-1} \int_{u \mid x}^{1} P(x \tau) T(x \tau) d \chi(\tau), \quad(x \text { real })
$$

must exist and be finite for every function $T(t)$ which is continuous in $[u, \rho)$, vanishes at its left end and has a finite limit $T(\rho-0)$.

(b) If in addition $P \subseteq Q$ then $\gamma(T)=T(\rho-0)$ for every such a function.

Proof. Substituting $r=u$ in Lemma 3.1.4 we infer the existence of a sequence of sequences $s^{(l)} \in c_{p}(l=1,2, \cdots)$ which satisfy $\lim _{p} s^{(l)}=$ $T(\rho-0)$ and

$$
\left\{\begin{array}{l}
s_{0}^{(l)}=s_{1}^{(l)}=\cdots=s_{m-1}^{(l)}=0, \quad \sum_{k=0}^{\infty}\left|p_{k} s_{k}^{(l)}\right| u^{k}<1 / l \\
\left|T_{p}\left(s^{(l)}, x\right)-T(x)\right|<1 / l, \quad(u \leqq x<\rho, l=1,2, \cdots) .
\end{array}\right.
$$

By (3.1.8)

$$
T_{q}\left(s^{(l)}, x\right)=[Q(x)]^{-1} \int_{u \mid x}^{1} P(x \tau) T_{p}\left(s^{(l)}, x \tau\right) d \chi(\tau)+\sum_{k=0}^{\infty} e_{x k} s_{k}^{(l)} .
$$

We denote $\lim _{q} s^{(l)}=T_{q}\left(s^{(l)}, \rho-0\right)=\beta_{l}$ (the limit exists since $s^{(l)} \in$ $c_{p} \subseteq c_{q}$ ). From (3.1.9) and (3.1.10) we have, then,

$$
\begin{aligned}
& \varlimsup_{x \rightarrow 0^{-}}\left|[Q(x)]^{-1} \int_{u \mid x}^{1} P(x \tau) T(x \tau) d \chi(\tau)-\beta_{l}\right| \\
& \leqq \varlimsup_{x \rightarrow \rho^{-}}|Q(x)|^{-1} \int_{u / x}^{1}|P(x \tau)| l^{-1}|d \chi(\tau)|+\varlimsup_{x \rightarrow \rho^{-}} \sum_{k=0}^{\infty}\left|e_{x k} s_{k}^{(l)}\right| \\
& \leqq R / l+\sum_{k=0}^{\infty} R\left|p_{k} s_{k}^{(l)}\right| u^{k} \leqq 2 R / l .
\end{aligned}
$$

From this it is easily deduced that $\left\{\beta_{l}\right\}$ is a Cauchy sequence-hence convergent. Denoting its limit by $\beta$ we have

$$
\varlimsup_{x \rightarrow \rho-}\left|[Q(x)]^{-1} \int_{u \mid x}^{1} P(x \tau) T(x \tau) d \chi(\tau)-\beta\right| \leqq 2 R / l+\left|\beta_{l}-\beta\right|
$$

which yields, with $l \rightarrow \infty, \gamma(T)=\beta$.

This completes the proof of part (a). Part (b) is proved in a 
very similar way. Only this time, since $s^{(l)} \in c_{p}, P \subseteq Q$, we have

$$
\beta_{l}=\lim _{q} s^{(l)}=\lim _{p} s^{(l)}=T(\rho-0) ; \text { so } \gamma(T)=\beta=T(\rho-0) .
$$

3.1.14. Proof of Theorem 2.2.7. By Remark 3.1.9 we may consider the case $r_{p q}=1, \rho_{q}=\rho_{p}=\rho, \alpha_{q}=\alpha_{p}=\alpha$ only. Assume, first, that $c_{p} \subseteq c_{q}$ and that the limit $1 / Q\left(\rho_{q}-0\right)$ does not exist. We have to show that $Q(x)=\kappa P(x)$ :

Let $u<t_{0}<t_{1}<\rho$ and let $h(t)$ be some continuous function in $[u, \rho)$, which vanishes in $\left[u, t_{0}\right]$ and in $\left[t_{1}, \rho\right)$. Denote $T(t)=h(t) / P(t)$. From Lemma 3.1.13 (a) we infer the existence and finiteness of the limit

$$
\gamma(T)=\lim _{x \rightarrow \rho_{-}}[Q(x)]^{-1} \int_{t=u}^{x} P(t) T(t) d \chi(t / x)=\lim _{x \rightarrow \rho_{-}}[Q(x)]^{-1} \int_{t=t_{0}}^{t_{1}} h(t) d \chi(t / x) .
$$

Denoting

$$
\chi_{x}(t)=\chi(t / x), \quad\left(t_{0} \leqq t \leqq x, t_{1}<x<\rho\right)
$$

we get

$$
\gamma(T)=\lim _{x \rightarrow \rho-}[Q(x)]^{-1} \int_{t_{0}}^{t_{1}} h(t) d \chi_{x}(t) .
$$

The functions $\chi_{x}(t)\left(t_{1} \leqq x<\rho\right)$ have uniformly bounded variation in $\left[t_{0}, t_{1}\right]$. This is because denoting $u \rho / t_{0}=y$ we have $u<y<\rho$ and

$$
\begin{array}{r}
\int_{t_{0}}^{t_{1}}\left|d \chi_{x}(t)\right|=\int_{t=t_{0}}^{t_{1}}|d \chi(t / x)| \leqq \int_{t_{0} \mid x}^{1}|d \chi(\tau)| \leqq \int_{u / y}^{1}|d \chi(\tau)|<\infty, \\
\quad\left(t_{1} \leqq x<\rho\right) .
\end{array}
$$

Therefore the following limit exists (see Widder [17] Theorem 16.4 Ch. I):

$$
\lim _{x \rightarrow \rho_{-}} \int_{t_{0}}^{t_{1}} h(t) d \chi_{x}(t)=\int_{t_{0}}^{t_{1}} h(t) d\left[\lim _{x \rightarrow \rho-} \chi_{x}(t)\right]=\int_{t_{0}}^{t_{1}} h(t) d \chi(t / \rho) .
$$

Since $1 / Q(x)$ does not converge to a finite limit, as $x \rightarrow \rho^{-}$, we infer from the existence and finiteness of $\gamma(T)$ (see (3.1.14)) that

$$
\int_{t_{0}}^{t_{1}} h(t) d \chi(t / \rho)=0 \text {. }
$$

The argument which led to this result is correct for every function $h(t)$, which is continuous in $\left[t_{0}, t_{1}\right]$ and vanishes at both its ends. Hence $\chi(t / \rho)=$ const. for $t \in\left(t_{0}, t_{1}\right)$. Since $t_{0}$ and $t_{1}$ may be taken as close as one wishes to $u$ and $\rho$, respectively, we actually have $\chi(\tau)=$ const. for $\tau \in(u / \rho, 1)$. Hence, by (3.1.8) 


$$
\begin{aligned}
& q_{k}=\kappa p_{k}+Q(x) e_{x k} / x^{k}, \quad \kappa=\chi(1)-\chi(1-0) \\
&(u \leqq x<\rho, k=0,1, \cdots)
\end{aligned}
$$

which proves that $Q(x) e_{x k} / x^{k}$ is independent of $x$. We may therefore write

$$
\left\{\begin{array}{l}
q_{k}=\kappa p_{k}+q_{k}^{\prime}, \quad(k=0,1, \cdots) \\
\text { where by }(3.1 .10), \\
\left|q_{k}^{\prime} x^{k} / Q(x)\right| \leqq R\left|p_{k}\right| u^{k}, \quad(u \leqq x<\rho, k=m, m+1, \cdots) .
\end{array}\right.
$$

From this it follows immediately that $\kappa \neq 0$ (otherwise we would have $r_{p q}<1$ ).

We complete the proof by showing that $q_{k}^{\prime}=0(k=0,1, \cdots)$ :

Let $i \in\{0,1, \cdots\}$ and choose $r=u$,

$$
T(x)= \begin{cases}p_{i} r^{i} / p(r), & x=r \\ \text { linear } & , \quad r \leqq x \leqq r_{1} ; \quad r<r_{1}<\rho \\ 0 & , \quad r_{1} \leqq x<\rho\end{cases}
$$

in Lemma 3.1.4. We infer the existence of a sequence of sequences, $s^{(l)} \in c_{p}^{(0)}(l=1,2, \cdots)$, such that

$$
\left\{\begin{array}{l}
s_{j}^{(l)}=0,(j \neq i, j<\max \{i, m\}), \quad s_{i}^{(l)}=1 \\
\sum_{k=2+1}^{\infty}\left|p_{k} s_{l k}^{(l)}\right| u^{k}<1 / l, \quad\left|T_{p}\left(s^{(l)}, x\right)\right|<\delta(x) / l \quad\left(r_{1} \leqq x<\rho\right) .
\end{array}\right.
$$

From (3.1.15) we have

$$
T_{q}\left(s^{(l)}, x\right)=\kappa[P(x) / Q(x)] T_{p}\left(s^{(l)}, x\right)+q_{2}^{\prime} x^{i} / Q(x)+\sum_{k=i+1}^{\infty} q_{k}^{\prime} s_{k}^{(l)} x^{k} / Q(x)
$$

so, for $r_{1} \leqq x<\rho$ we get from (3.1.15) and (3.1.16)

$$
\begin{aligned}
\left|q_{2}^{\prime} x^{i} / Q(x)-T_{q}\left(s^{(l)}, x\right)\right| & \leqq\left|\kappa[P(x) / Q(x)] T_{p}\left(s^{(l)}, x\right)\right|+\sum_{k=i+1}^{\infty}\left|q_{k}^{\prime} s_{k}^{(l)}\right| x^{k} /|Q(x)| \\
& \leqq|\kappa P(x) / Q(x)| \delta(x) / l+R / l .
\end{aligned}
$$

Choosing properly $\delta(x)>0$ (e.g. $\delta(x)=|Q(x) / P(x)|$ ) we may then get, $\left|q_{i}^{\prime} x^{i} / Q(x)-T_{q}\left(s^{(l)}, x\right)\right|<M / l, \quad M<\infty, \quad\left(r_{1} \leqq x<\rho, l=1,2, \cdots\right)$.

Since $s^{(l)} \in c_{p}^{(0)} \subseteq c_{q}$ the limits $\beta_{l}=\lim _{x \rightarrow \rho_{-}} T_{q}\left(s^{(l)}, x\right)(l=1,2, \cdots)$ all exist and we get

$$
\varlimsup_{x \rightarrow \rho_{-}}\left|q_{2}^{\prime} x^{i} / Q(x)-\beta_{l}\right| \leqq M / l \quad(l=1,2, \cdots) .
$$

This implies that $\beta_{l}$ is a Cauchy sequence, thus having a limit $\beta$. Obviously 


$$
\varlimsup_{x \rightarrow \rho_{-}}\left|q_{i}^{\prime} x^{i} / Q(x)-\beta\right| \leqq M / l+\left|\beta_{l}-\beta\right|
$$

so letting $l$ tend to $\infty$ we infer the existence of the limit $\lim _{x \rightarrow 0-} q_{i}^{\prime} x^{i} / Q(x)=\beta$, which is impossible, since $1 / Q(\rho-0)$ does not exist, unless $q_{i}^{\prime}=0$. This completes the proof for the case $c_{p} \subseteq c_{q}$.

The proof for the case $P \subseteq Q$ is similar. We just have to use Lemma 3.1.13 (b) instead of (a) to infer that $\gamma(T)=0$, and then remember that $s^{(l)} \in c_{p}^{(0)} \subseteq c_{q}^{(0)}$, so $\beta_{l}=0(l=1,2, \cdots)$, which means that $\beta=0$ and therefore $q_{i}^{\prime}=0$, unless $1 / Q(\rho-0)=0$.

3.1.15. Proof of Remark 2.2.8. First let us show the existence of two entire functions $P(x)=\sum p_{k} x^{k}, Q(x)=\sum q_{k} x^{k}$ which satisfy

( a ) $Q(x)=\left(\int_{0}^{x} P(t) d t\right) / x=\sum_{k=0}^{\infty} p_{k} x^{k} /(k+1),(0<x<+\infty)$

(b) $P(x) \geqq 1,(0 \leqq x<+\infty)$

(c) The limit $1 / Q(+\infty)$ does not exist.

In order to prove this, notice that the function

$$
f(x)=\int_{0}^{x}\left[\left(1-e^{-t}\right) / t\right] d t=x / 1 \cdot 1 !-x^{2} / 2 \cdot 2 !+x^{3} / 3 \cdot 3 !-\cdots
$$

is entire and satisfies $f(+\infty)=+\infty$. Hence the function $Q(x)=3+$ $\sin [\theta f(x)]$ (where $0<\theta \leqq 1$ is a constant to be specified later) is also entire and clearly satisfies (c). The relation (a), then, defines $P(x)$ to be:

$$
P(x)=[x Q(x)]^{\prime}=3+\sin [\theta f(x)]+\theta\left(1-e^{-x}\right) \cos [\theta f(x)],
$$

which is obviously an entire function that satisfies (b). Inserting the power series expansion of $f(x)$ into the expansion of the sin, in order to obtain the expansion of $Q(x)$, we see that each of the coefficients $q_{k}$ is a polynomial in $\theta$ which is not the null polynomial. One may choose $\theta$ to differ from all of the roots of these polynomials and get $q_{k} \neq 0(k=0,1, \cdots)$. It follows, then, from (a), that (2.1.1) is satisfied also.

Now, from (a) it follows that

$$
T_{q}(s, x)=\left[\int_{0}^{x} P(t) T_{p}(s, t) d t\right] /\left[\int_{0}^{x} P(t) d t\right], \quad(0<x<+\infty) .
$$

Hence, by (b), $P \subseteq Q$. This is so although (c) is satisfied.

It should be noticed that, by (a) and Theorem 2.2.4 (iii), $Q \nsubseteq P$ so $P$ and $Q$ are essentially different power methods.

Examples of pairs of power methods, $P \cong Q$ for which $1 / Q(+\infty) \neq 0$, can be easily constructed by Theorem 2.3.10. 
LEMMA 3.1.16. denote

(a) If in Lemma 3.1 .10 we add the assumption $c_{p} \subseteq c_{q}$ and

$$
e_{x k}^{\prime}=e_{x k}-\left[p_{k} u^{k} / P(u)\right] \sum_{i=0}^{\infty} e_{x i}, \quad(u \leqq x<\rho, k=0,1, \cdots)
$$

then the limits

$$
e_{k}^{\prime}=\lim _{x \rightarrow \beta-} e_{x k}^{\prime} \quad(x \text { real, } k=0,1, \cdots)
$$

all exist and are finite. (b) If, in addition, $P \subseteq Q$ then for all $k \in\{0,1, \cdots\} \quad e_{k}^{\prime}=0$.

Proof. First, we notice that by (3.1.10) and (3.1.17)

$$
\begin{cases}\left|e_{x k}^{\prime}\right| \leqq R^{\prime}\left|p_{k}\right| u^{k}, & (u \leqq x<\rho, k=m, m+1, \cdots) \\ \left|e_{x k}^{\prime}\right| \leqq R^{\prime} & , \quad(u \leqq x<\rho, k=0,1, \cdots)\end{cases}
$$

Next, we see from (3.1.8) that

$$
Q(x)=\int_{u \mid x}^{1} P(x \tau) d \chi(\tau)+Q(x) \sum_{k=0}^{\infty} e_{x k},
$$

so,

$$
\sum_{i=0}^{\infty} e_{x \imath}=1-[Q(x)]^{-1} \int_{u / x}^{1} P(x \tau) d \chi(\tau) .
$$

Substituting this into (3.1.17) and using (3.1.8) we get for every $s \in c_{p}$ :

$$
\begin{aligned}
T_{q}(s, x)= & T_{p}(s, u)+[Q(x)]^{-1} \int_{u \mid x}^{1} P(x \tau)\left[T_{p}(s, x \tau)\right. \\
& \left.-T_{p}(s, u)\right] d \chi(\tau)+\sum_{k=0}^{\infty} e_{x k}^{\prime} s_{k} .
\end{aligned}
$$

Let now $i \in\{0,1, \cdots\}$ and choose in Lemma 3.1.4 $r=u$ and $T(x) \equiv$ $p_{i} u^{i} / P(u)$. From Lemma 3.1.4 we infer the existence of a sequence of sequences $s^{(l)} \in c_{p}(l=1,2, \cdots)$, that satisfy

$$
s_{j}^{(l)}=0 \quad(j \neq i, j<\max \{i, m\}), \quad s_{\imath}^{(l)}=1, \quad \sum_{k=\imath+1}^{\infty}\left|p_{k} s_{k}^{(l)}\right| u^{k}<1 / l .
$$

Since $s^{(l)} \in c_{p} \subseteq c_{q}$ we infer, from (3.1.20) with the aid of Lemma 3.1.13(a), the existence and finiteness of the limits

$$
\beta_{l}=\lim _{x \rightarrow \rho^{-}} \sum_{k=0}^{\infty} e_{x k}^{\prime} s_{k}^{(l)} \quad(l=1,2, \cdots) .
$$

By (3.1.19) 


$$
\left|e_{x i}^{\prime}-\sum_{k=0}^{\infty} e_{x k}^{\prime} s_{k}^{(l)}\right|=\left|-\sum_{k=i+1}^{\infty} e_{x k}^{\prime} s_{k}^{(l)}\right| \leqq R^{\prime} / l
$$

Therefore

$$
\varlimsup_{x \rightarrow \rho-}\left|e_{x i}^{\prime}-\beta_{l}\right| \leqq R^{\prime} / l
$$

From this it follows that $\beta_{l}$ is a Cauchy sequence. Denoting its limit by $\beta$ we get then,

$$
\varlimsup_{x \rightarrow \rho_{-}}\left|e_{x i}^{\prime}-\beta\right| \leqq R^{\prime} / l+\left|\beta-\beta_{l}\right|,
$$

which yields, with $l \rightarrow \infty$, that actually $e_{i}^{\prime}=\beta$.

Thus (a) is proved. The proof of (b) is similar. We just have to notice that in case $P \subseteq Q$ we get from (3.1.20) and from Lemma 3.1.13(b) that $\beta_{l}=0(l=1,2, \cdots)$. Hence $e_{i}^{\prime}=\beta=0$.

3.1.17. Proof of Theorem 2.2.9. This is an immediate consequence of Lemmas 3.1.10, 3.1.13, and 3.1.16.

3.1.18. Proof of Remark 2.2.10. The fact that $\phi$ is not constant in $[1-\delta, 1]$ follows immediately from the definition of $r_{p q}$ (see $\S 2.2 .3)$. The uniqueness of $\phi$ in certain intervals follows from Trautner's theorem (see $\S 3.1 .2$ ).

3.1.19. Proof of Theorem 2.2.11. It is sufficient to consider the case $r_{p q}=1, \rho_{q}=\rho_{p}=\rho, \alpha_{q}=\alpha_{p}=\alpha$ and to show that $c_{p}^{(0)} \subseteq c_{q}^{(0)}$. By (2.2.1) we may write

$$
q_{k}=p_{k}\left(\int_{0}^{1} \tau^{k} d \phi(\tau)+\theta_{k}\right)+q_{k}^{\prime}, \quad\left|\theta_{k}\right| \leqq R \theta^{k} \quad(k=0,1, \cdots)
$$

where $q_{k}^{\prime}=0$ for $k \geqq m$. Hence for every $s \in c_{p}^{(0)}$

$$
\begin{aligned}
T_{q}(s, x)= & {[Q(x)]^{-1} \int_{0}^{1} P(x \tau) T_{p}(s, x \tau) d \phi(\tau)+[Q(x)]^{-1} \sum_{k=0}^{\infty} \theta_{k} p_{k} s_{k} x^{k} } \\
& +[Q(x)]^{-1} \sum_{k=0}^{m-1} q_{k}^{\prime} s_{k} x^{k}=\sigma_{1}(x)+\sigma_{2}(x)+\sigma_{3}(x) .
\end{aligned}
$$

It easily follows that $\sigma_{1}(x) \rightarrow 0, \sigma_{2}(x) \rightarrow 0, \sigma_{3}(x) \rightarrow 0 \quad(x \rightarrow \rho-)$ for every $s \in c_{p}^{(0)}$; so $T_{q}(s, x) \rightarrow 0$, which completes the proof.

3.1.20. Proof of Theorem 2.2.13. It is sufficient to consider the case $\rho_{q}=\rho_{p}=\infty, r_{p q}=1, \alpha_{q}=\alpha_{p}=\alpha$. The proof is based on (3.1.19) and (3.1.20), which are deduced here as in section 3.1.16.

It should be observed that (3.1.19) implies: 


$$
\lim _{x \rightarrow+\infty} \sum_{k=0}^{\infty} e_{x k}^{\prime} s_{k}=\sum_{k=0}^{\infty}\left(\lim _{x \rightarrow+\infty} e_{x k}^{\prime}\right) s_{k}=\sum_{k=0}^{\infty} e_{k}^{\prime} s_{k}
$$

3.1.21. Proof of Remark 2.2.14. Follows immediately from the proofs of Theorems 2.2.11 and 2.2.13.

3.2. Proofs of the results in Section 2.3.

3.2.1. Proof of Theorem 2.3.2. This follows in a straightforward way from Theorem III of Włodarski [19].

LEMMA 3.2.2. If $P$ is a regular power method with $\rho_{p}=\infty$ then $P(\varepsilon x) / P(x) \rightarrow 0(x \rightarrow+\infty, x$ real $)$ for every $0<\varepsilon<1$.

Proof. If $0<\varepsilon<1$ then $\varepsilon^{k} \rightarrow 0(k \rightarrow \infty)$. Hence the regularity of $P$ yields

$$
\lim _{x \rightarrow+\infty} P(\varepsilon x) / P(x)=\lim _{x \rightarrow+\infty} \sum_{k=0}^{\infty} p_{k} \varepsilon^{k} x^{k} / P(x)=\lim _{p}\left\{\varepsilon^{k}\right\}=0 .
$$

3.2.3. Proof of Theorem 2.3.4. Assume first that $P$ is conservative. We define $s_{j}=0$ if $j \in J$ (see Remark 2.2.2), $s_{j}=q_{j} /\left(p_{j} r_{p q}^{j}\right)$ if $p_{j} \neq 0$ and $s_{j}=\lim q_{k} /\left(p_{k} r_{p q}^{k}\right)$ if $p_{j}=0, j \notin J$. From Theorem 2.2.4 (iii) we see that the sequence $s=\left\{s_{k}\right\}$ is well defined and convergent. Now,

$$
\begin{aligned}
Q(x) / P\left(x r_{p q}\right)= & {\left[P\left(x r_{p q}\right)\right]^{-1} \sum_{k=0}^{\infty} q_{k} x^{k}=\left[P\left(x r_{p q}\right)\right]^{-1} \sum_{k=0}^{\infty} p_{k} s_{k}\left(x r_{p q}\right)^{k} } \\
& +\left[P\left(x r_{p q}\right)\right]^{-1} \sum_{k \in J} q_{k} x^{k}=T_{p}\left(s, x r_{p q}\right)+\sigma(x)
\end{aligned}
$$

By Theorem 2.3.2 $\lim _{x \rightarrow \rho_{q}-} \sigma(x)$ exists, and from Theorem 2.2.4, and since $P$ is conservative, $\lim _{x \rightarrow \rho_{q^{-}}} T_{p}\left(s, x r_{p q}\right)$ also exists. This establishes the existence of $\lim Q(x) / P\left(x r_{p q}\right)$.

Now assume that $\rho_{p}=\infty$ and $P$ is regular. We may write

$$
Q(x) / P(r x)=\left[Q(x) / P\left(r_{p q} x\right)\right] \cdot\left[P\left(r_{p q} x\right) / P(r x)\right]
$$

and this, by Lemma 3.2.2, tends to zero in case $r>r_{p q}$.

If $0<r<r_{p q}$ we may choose $\tau_{0} \in\left(r / r_{p q}, 1\right)$ and have, by Theorems 2.2.9 and 2.3.2, for $x \rightarrow+\infty$,

$$
\begin{aligned}
O(1)= & |Q(x)|^{-1} \int_{\tau_{0}}^{1}\left|P\left(x r_{p q} \tau\right)\right||d \dot{\phi}(\tau)| \geqq L|Q(x)|^{-1} \int_{\tau_{0}}^{1} \hat{P}\left(x r_{p q} \tau\right)|d \phi(\tau)| \\
& \geqq L\left|P\left(x r_{p q} \tau_{0}\right) / Q(x)\right| \int_{\tau_{0}}^{1}|d \dot{\phi}(\tau)|
\end{aligned}
$$

From Remark 2.2.10 $\int_{\tau_{0}}^{1}|d \phi(\tau)| \neq 0$; so $P\left(x r_{p q} \tau_{0}\right) / Q(x)=O(1)(x \rightarrow+\infty)$. 
From Lemma 3.2.2 we have then,

$$
P(r x) / Q(x)=\left[P\left(x r_{p q} \tau_{0}\right) / Q(x)\right] \cdot\left[P(r x) / P\left(x r_{p q} \tau_{0}\right)\right] \rightarrow 0 \quad(x \rightarrow+\infty)
$$

which completes the proof.

LEMma 3.2.4. Let $E$ be an $F K$ space and let $\left\{f_{n}\right\}$ be a sequence of continuous linear functionals on $E$. If $\lim _{n \rightarrow \infty} f_{n}(s)$ exists for all terms $s$ of some fundamental set in $E$ and if the sequence $\left\{f_{n}(s)\right\}$ is bounded for every $s \in E$ then the limit $f(s)=\lim _{n \rightarrow \infty} f_{n}(s)$ exists for every $s \in E$ and is a continuous linear functional in $E$.

Proof. For a proof of a more general theorem, see Dunford \& Schwartz [9] II 1.18.

3.2.5. Proof of Theorem 2.3.5 It is known that $c_{p}$ is an $F K$ space (see [2] and [21]), and it was proved in [21] that under the conditions of Theorem 2.3.5 $U$ (see $\S 2.3 .6$ ) is fundamental in $c_{p}$. From Lemma 3.2.4, it is sufficient then to show that $c_{p} \subseteq m_{q}$ and that $\lim _{q} s$ exists for every $s \in U$. But the existence of these limits follows from the existence of $1 / Q\left(\rho_{q}-0\right)$. The inclusion $c_{p} \subseteq m_{q}$ follows from (2.2.2) and from the boundedness of $1 / Q(x)$, after expressing $T_{q}(s, x)$ in terms of $T_{p}(s, x)$, via (2.2.1), as it was done at the beginning of $\S 3.1 .19$.

3.2.6. Proof of Theorem 2.3.7. If $U$ is fundamental in $c_{p}$, then the proof is based on Lemma 3.2.4 in the same way as Theorem 2.3.5 (see $\S 3.2 .5$ ). This is possible because (i), (ii), (iii) imply $c_{p} \leqq m_{q}$, as is easily seen from the identity

$$
T_{q}(s, x)=[Q(x)]^{-1} \int_{u_{1} x}^{1} P\left(x r_{p q} \tau\right) T_{p}\left(s, x r_{p q} \tau\right) d \phi(\tau)+\sum_{k=0}^{\infty} e_{x k} s_{k},
$$

which follows from (i). Also, the boundedness of

$$
T_{q}\left(e^{(j+l)}, x\right)=q_{j+l} x^{j+l} / Q(x) \quad\left(\alpha_{q} \leqq x<+\infty\right)
$$

implies, when taking $l \geqq 1$, such that $q_{j+l} \neq 0$ (see Remark 2.2.1), that $x^{j} / Q(x) \rightarrow 0(x \rightarrow+\infty)$ for all $j$. So $\lim _{q} e^{(j)}=0$ for $j=0,1, \cdots$ (in a similar way $\lim _{p} e^{(j)}=0$ ).

Thus $U \subseteq c_{q}$ and Lemma 3.2.4 yields $c_{p} \subseteq c_{q}$. The consistency of $P$ and $Q$ follows now because the continuous linear functionals $\lim _{p}, \lim _{q}$ coincide on the fundamental set $U$, hence all over $c_{p}$.

Consider now the case that $Q(\varepsilon x) / Q(x) \rightarrow 0$ and assume, for the sake of simplicity, that $r_{p q}=1$. We define $e_{x k}(v)$ by

$$
\begin{aligned}
q_{k}=p_{k} \int_{v / x}^{1} \tau^{k} d \phi(\tau) & +Q(x) e_{x k}(v) / x^{k}, \\
& (u \leqq v \leqq x<+\infty, k=0,1, \cdots) .
\end{aligned}
$$


From (ii) we infer the existence of an $M$, independent of both $v$ and $x$, such that

$$
\text { (ii })^{*} \quad|Q(x)|^{-1} \int_{v / x}^{1}|P(x \tau)||d \phi(\tau)| \leqq M \quad(u \leqq v \leqq x<+\infty) .
$$

Comparing (i) and (i)* and assuming $v / x=u / y$ we get

$$
Q(x) e_{x k}(v) / x^{k}=Q(y) e_{y k} / y^{k} \Longrightarrow\left|e_{x k}(v)\right| \leqq|Q(u x / v) / Q(x)|(v / u)^{k}\left|e_{y k}\right|,
$$

which implies, by (iii), for every sufficiently large $v$,

(iii)*

$$
\begin{cases}\left|e_{x k}(v)\right| \leqq M_{v}\left|p_{k}\right| v^{k} & (v \leqq x<+\infty, k=m, m+1, \cdots) \\ \left|e_{x k}(v)\right| \leqq M_{v} & (v \leqq x<+\infty, k=0,1, \cdots)\end{cases}
$$

where $M_{v}$ is independent of $x$. Also, for sufficiently large $v$

(iv)*

$$
\lim _{x \rightarrow+\infty} e_{x k}(v)=0 \quad(x \text { real, } k=0,1, \cdots) .
$$

Now, from (i)* we have

$$
\begin{aligned}
T_{q}(s, x) & =[Q(x)]^{-1} \int_{v \mid x}^{1} P(x \tau) T_{p}(s, x \tau) d \phi(\tau)+\sum_{k=0}^{\infty} e_{x k}(v) s_{k} \\
& =\sigma_{1}(v, x)+\sigma_{2}(v, x) .
\end{aligned}
$$

From (ii)* we have

$$
\sup _{x \geqq X}\left|\sigma_{1}(v, x)\right| \leqq M \sup _{y \geqq v}\left|T_{p}(s, y)\right|
$$

for all $X \geqq v$, and from (iii)*, (iv)*, for every sufficiently large $v$ we have

$$
\lim _{x \rightarrow+\infty}\left|\sigma_{2}(v, x)\right|=0 .
$$

Hence for each $v$ sufficiently large,

$$
\varlimsup_{x \rightarrow+\infty}\left|T_{q}(s, x)\right| \leqq M \sup _{y \geq v}\left|T_{p}(s, y)\right| .
$$

Thus, if $s \in c_{p}^{(0)}$, it follows immediately that $\lim _{q} s=0$. Therefore, $c_{p}^{(0)} \subseteq c_{q}^{(0)}$ which implies $P \subseteq Q$.

3.2.7. Proof of Remark 2.3.8. This follows immediately from Lemma 3.2.2.

3.2.8. Proof of Theorem 2.3.10. The proof is immediate if we use the identities 


$$
\begin{aligned}
Q(x) & =\int_{0}^{1} P(x r \tau) d \phi(\tau) \\
T_{q}(s, x) & =[Q(x)]^{-1} \int_{0}^{1} P(x r \tau) T_{p}(s, x r \tau) d \phi(\tau), \quad\left(s \in c_{p}\right),
\end{aligned}
$$

which follow from (2.3.2). Actually we infer that

$$
\begin{aligned}
Q(+\infty)= & P(0)[\phi(0+)-\phi(0)]+[\phi(1)-\phi(0+)] P(+\infty) \\
\lim _{q} s= & {[Q(+\infty)]^{-1} P(0)[\phi(0+)-\phi(0)] s_{0} } \\
& +[Q(+\infty)]^{-1}[\phi(1)-\phi(0+)] P(+\infty) \lim _{p} s
\end{aligned}
$$

ACKNowledgement. The author wishes to thank Professor A. Jakimovski for his valuable advice during the preparation of this paper.

\section{REFERENCES}

1. A. Amir (Jakimovski), Some relations between the methods of summability of Abel, Borel, Cesàro, Hölder, and Hausdorff, J. Analyse Math., 3 (1953/54), 346-381.

2. A. Birkholic, On generalized power methods of limitation, Studia Math., 27 (1966), 213-245.

3. - Remarks on a moment problem and a problem of perfection of power methods of limitation, Colloq. Math., 24 (1971), 89-94.

4. D. Borwein, On Methods of summability based on power series, Proc. Roy. Soc., Edinburgh, 64 (1957), 342-349.

5. - On a scale of Abel-type summability methods, Proc. Cambridge Philos. Soc., 53 (1957), 318-322.

6. — A logarithmic method of summability, J. London Math. Soc., 33 (1958), $212-220$.

7. - On methods of summability based on integral functions, Proc. Cambridge Philos. Soc., 55 (1959), 23-30.

8. - On methods of summability based on integral functions, II. Proc. Cambridge Philos. Soc., 56 (1960), 125-131.

9. N. Dunford and J. T. Schwartz, Linear Operators, Part I (Interscience 1958).

10. G. H. Hardy, Divergent Series, (Oxford, 1949).

11. E. W. Hobson, The Theory of Functions of A Real Variable, Vol. I (Cambridge, 1927).

12. L. Hoischen, Some inclusion theorems for generalized Abel and Borel summability, J. Londoh Math. Soc., 42 (1967), 229-234.

13. M. Lazič, Sur les procédés fonctionnels (de limitation). Mat. Vesnik, 6 (21), (1969). 425-436.

14. J. G. Mikusinski, Remarks on the moment problem and a theorem of Picone, Colloq. Math., 2 (1951), 138-141.

15. C. Ryll-Nardzewski, The Borel method is perfect, Bull. Acad. Polon. Sci. Sér., Sci. Math. Astronom. Phys. 10 (1962), 469-650.

16. R. Trautner, Density properties of Hausdorff moment sequences, Tôhoku Math. J. 24 (1972), 347-352.

17. D. V. Widder, The Laplace Transform, (Oxford, 1946).

18. A. Wilansky, Functional Analysis, (Blaisdell 1964).

19. L. Włodarski, Sur les méthodes continue de limitation (I), Studia Math., 14 (1954), 161-187 (1955). 
20. K. Zeller, Sur la méthode de sommation d'Abel, C. R. Acad. Sci. Paris, 236 (1953), 568-569.

21. A. Ziv, Inclusion relations between power methods and matrix methods of limitation, Trans. Amer. Math. Soc., (to appear).

22. - Rate of growth and convergence factors for power methods of limitation, Proc. Cambridge Philos. Soc., 76 (1974), 241-246.

Received December 5, 1974 and in revised form November 9, 1975. This research was carried out while the author was a candidate for his $\mathrm{Ph}$. D. at the Faculty of Mathematics, Technion, Israel Institute of Technology, Haifa, Israel.

IBM IsRael Scientific Center TeChNion City

HAIFA, ISRAEL 



\section{PACIFIC JOURNAL OF MATHEMATICS}

EDITORS

RICHARD ARENS (Managing Editor)

University of California

Los Angeles, California 90024

R. A. BEAUMONT

University of Washington

Seattle, Washington 98105

J. DugundjI

Department of Mathematics

University of Southern California

Los Angeles, California 90007

D. Gilbarg and J. Milgram

Stanford University

Stanford, California 94305

\section{ASSOCIATE EDITORS}

E. F. BeCKENBACH

B. H. NeUMaNN

F. WOLF

K. YoSHIDA

\section{SUPPORTING INSTITUTIONS}

UNIVERSITY OF BRITISH COLUMBIA

CALIFORNIA INSTITUTE OF TECHNOLOGY

UNIVERSITY OF CALIFORNIA

MONTANA STATE UNIVERSITY

UNIVERSITY OF NEVADA

NEW MEXICO STATE UNIVERSITY

OREGON STATE UNIVERSITY

UNIVERSITY OF OREGON

OSAKA UNIVERSITY

\author{
UNIVERSITY OF SOUTHERN CALIFORNIA \\ STANFORD UNIVERSITY \\ UNIVERSITY OF TOKYO \\ UNIVERSITY OF UTAH \\ WASHINGTON STATE UNIVERSITY \\ UNIVERSITY OF WASHINGTON \\ AMERICAN MATHEMATICAL SOCIETY \\ NAVAL WEAPONS CENTER
}




\section{Pacific Journal of Mathematics \\ Vol. 67, No. $1 \quad$ January, 1976}

Gregory Wayne Brumfiel and John W. Morgan, Homotopy theoretic

consequences of $N$. Levitt's obstruction theory to transversality for

spherical fibrations . ................................ 1

Jacob Burbea, Total positivity of certain reproducing kernels ........... 101

Wai-Mee Ching, The structure of standard $C^{*}$-algebras and their

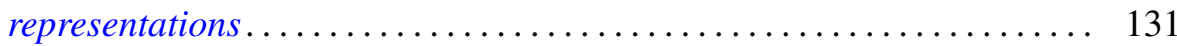

Satya Deo, The cohomological dimension of an $n$-manifold is $n+1 \ldots \ldots 155$

Masahiko Fujiwara and Masaki Sudo, Some forms of odd degree for which the Hasse principle fails ................................. 161

Mikihiro Hayashi, Smoothness of analytic functions at boundary points ... 171

Rebecca A. Herb, A uniqueness theorem for tempered invariant

eigendistributions ................................ 203

David Alan Legg, Orlicz space convergence of martingales of

Radon-Nikodým derivatives given a $\sigma$-lattice.................. 209

D. B. McAlister, v-prehomomorphisms on inverse semigroups......... 215

Bruno J. Mueller, Localization in fully bounded Noetherian rings . ........ 233

Donald J. Newman and A. R. Reddy, Rational approximation to $x^{n} \ldots \ldots .247$

Abraham Ziv, Inclusion relations between power methods of limitation.... 251 